\title{
APOYO COMUNITARIO PERCIBIDO EN JÓVENES Y VOLUNTARIADO
}

\author{
Rafael López Cordero \\ Departamento de Didáctica de las Ciencias Sociales \\ Universidad de Granada \\ raloco@ugr.es \\ Lucía Herrera Torres \\ Departamento de Psicología Evolutiva y de la Educación \\ Universidad de Granada \\ luciaht@ugr.es
}

Fecha de Recepción: 7 Abril 2019

Fecha de Admisión: 30 Abril 2019

\section{RESUMEN}

El apoyo social comunitario se concibe como la ayuda entre las personas que comparten hogares, escuelas, vecindarios, lugares de trabajo, organizaciones y otros entornos comunitarios. El presente estudio se centra en la percepción que una muestra de jóvenes melillenses presenta sobre el apoyo social de su comunidad en función de si se participa o no en actividades de voluntariado. Para ello, se desarrolló un estudio de tipo correlacional. Los principales resultados ponen de manifiesto una relación positiva entre la participación en actividades de voluntariado y el apoyo comunitario percibido relativo a los siguientes elementos: la percepción que poseen los jóvenes sobre la valoración positiva de sus opiniones en su comunidad, el sentimiento de pertenencia a la comunidad, el hecho de colaborar en organizaciones y asociaciones de la comunidad, realizar actividades en su comunidad, formar parte de grupos sociales o cívicos, responder a las llamadas de apoyo en su comunidad, así como ser capaz de animar a los demás y lograr un mejor estado de ánimo. Por su parte, la relación es negativa con la no realización de actividades socio-recreativas en la comunidad. Se discute la necesidad de fomentar actividades de voluntariado entre los jóvenes, lo cual repercutirá en su bienestar e integración comunitaria.

Palabras clave: apoyo comunitario percibido; jóvenes; voluntariado

\section{ABSTRACT}

Perceived community support in young people and volunteering. Community social support is conceived as help among people who share homes, schools, neighborhoods, workplaces, organizations and other community settings. The present study focuses on the perception that a sample of young people in Melilla presents about the social support of their community depending on whether 


\section{APOYO COMUNITARIO PERCIBIDO EN JÓVENES Y VOLUNTARIADO}

or not they participate in volunteer activities. For this, a correlation study was developed. The main results show a positive relationship between participation in volunteer activities and perceived community support related to the following elements: the perception that young people have about their opinions being valued in their community, the feeling of belonging to the community, the fact of collaborating with community organizations and associations, carrying out activities in their community, being part of social or civic groups, responding to calls for support in their community, as well as being able to encourage others and get into a better mood. On the other hand, the relationship is negative with the non-performance of socio-recreational activities in the community. The need to promote volunteer activities among young people is discussed, which will affect their wellbeing and community integration.

Keywords: perceived community support; young people; volunteering

\section{INTRODUCCIÓN}

En el contexto de la Psicología Comunitaria se ha analizado el concepto y características del apoyo social comunitario (Moritsugu, Vera, Wong, \& Grover, 2016; Rappaport \& Seidman, 2000). Según Barrera (2010), el apoyo social comunitario hace referencia a la ayuda entre las personas que comparten hogares, escuelas, vecindarios, lugares de trabajo, organizaciones y otros entornos comunitarios. En su estudio se ha hallado relación con determinados elementos como el sentido comunitario, la vecindad y la integración social (Brissette, Cohen, \& Seeman, 2000; Farrel, Aubry, \& Coulombe, 2004).

Diferentes investigaciones relacionan la salud mental con el apoyo social comunitario (Lakey \& Orehek, 2011; McLaren \& Hawe, 2005), al igual que con el bienestar psicológico (Binder \& Freytag, 2013; Herrero, Meneses, Valiente, \& Rodríguez, 2004). En esta dirección, para Ng, Chan y Lai (2014) el apoyo comunitario es una variable mediadora de la satisfacción vital y la esperanza en niños de contextos deprimidos de Hong Kong. También se ha encontrado su repercusión positiva en procesos oncológicos, mediante el apoyo social, la expresión emocional y la resolución de problemas ofrecida por grupos comunitarios de apoyo al cáncer de mama (McDonald, Wall, Corwin, Brooks, \& Koopman, 2013). En adolescentes se trata de una factor de protección de los síntomas depresivos (Mancini, Rigoli, Heritage, Roberts, \& Piek, 2016; Patwardhan et al., 2017). Asimismo, se trata de un factor mediador de la potencial aparición de conflictos tanto en el ámbito del trabajo como familiar en trabajadores con bajos ingresos económicos (Kossek, Pichler, Meece, \& Barrat, 2008; Lambert, Casper, \& Eby, 2013). Igualmente, en personas mayores ejerce efectos positivos (Gadalla, 2010; Melchiorre et al., 2013). Así, por ejemplo, cuando dichas personas finalizan un periodo de ingreso hospitalario y perciben que tienen personas de apoyo al salir del hospital se incrementa la probabilidad de que no vuelvan a pasar por un nuevo proceso de hospitalización de forma inmediata frente a aquellas que no poseen dicha percepción (Coffey \& McCarthy, 2013).

En personas jóvenes también está asociado el apoyo social comunitario (ofrecido por familiares, amigos, vecinos, el contexto académico, etc.) con su satisfacción vital (Oberle, Schonert-Reichl, \& Zumbo, 2011). En estudiantes universitarios, el apoyo percibido en el contexto universitarios, principalmente procedente del profesorado, predice de forma significativa su rendimiento académico (Guthrie \& Fruiht, 2018). Tanto en jóvenes como adultos jóvenes se encuentra que las redes de apoyo social, las cuales incluyen a la familia, los amigos y el apoyo comunitario, se constituyen como fuertes variables predictoras del ajuste de personas del colectivo LGTB, incluyendo entre dicho ajuste la satisfacción con la vida, la autoestima y la estima LGTB (Snapp, Watson, Russell, Diaz, \& Ryan, 2015). La percepción del apoyo institucional también es un factor relevante para los jóvenes pertenecientes a poblaciones culturales y lingüísticas minoritarias (Esteban-Guitart, Viladot, \& Giles, 2015) 0 inmigrantes (Wang, Hsieh, Assari, Gaskin, \& Rost, 2018). 
Por otra parte, son diversos los trabajos en los que se ha explorado en los jóvenes su participación en actividades de voluntariado (Brewis, 2010; Hyde \& Knowles, 2013). Según Musick y Wilson (2007), el voluntariado es una conducta altruista cuya finalidad es ofrecer ayuda a otras personas, grupos, organizaciones, causas 0 a la comunidad en general, sin esperar ninguna recompensa a cambio. Linsay (2016), en un estudio de revisión de más de 1500 artículos sobre voluntariado, identificó desarrolla del capital humano (es decir, la experiencia práctica, la mejora de la autodeterminación, la autoconfianza, la capacidad de afrontamiento), potencia el capital social (es decir, habilidades sociales y de comunicación, la inclusión social) y favorece el capital cultural (es decir, la ayuda a los demás, la contribución a la comunidad). Esta autora también pone de manifiesto los beneficios del voluntariado en jóvenes con discapacidad.

En los jóvenes españoles, las principales causas de participación en actividades relacionadas con el voluntariado tienen que ver con la disposición de modelos familiares, especialmente en los padres, además del nivel de formación educativas de los últimos (García, Marcuello, \& Saz, 2015). Gil, Marcuello y Saz (2015) analizaron la participación en el voluntariado en 20 países de Europa. Entre los principales resultados hallados se pone de manifiesto que las características socioeconómicas pueden ser relevantes para que los jóvenes decidan participar en actividades de voluntariado pero que más determinantes son el formar parte de grupos en asociaciones no gubernamentales así como las actitudes que se posean ante diferentes ámbitos de la vida, como el familiar, laboral, religioso... Igualmente, el voluntariado y la salud se relacionan con el apoyo social percibido, tal y como indican Kumar, Calvo, Avendano, Sivaramakrishnan y Berkman (2012) en el estudio que realizaron que implicaba a 139 países.

Normalmente, en el desarrollo del voluntariado participan personas, de modo individual, o en el seno de asociaciones, ya sean laicas o religiosas. En este último caso, la religiosidad se ha relacionado, de forma habitual, con la implicación en diferentes acciones de voluntariado (Lewis, MacGregor, \& Putnam, 2013; Paxton, Reith, \& Glaville, 2014; Stroope, 2011). Sin embargo, la conducta prosocial es la que destaca dentro del voluntariado con mayor fuerza, lo cual se vincula al sentido de pertenencia a un grupo y ello tiene lugar tanto en grupos laicos como religiosos (Galen, Sharp, \& McNulty, 2015).

En función de lo descrito previamente, el objetivo principal de este trabajo es analizar la percepción que una muestra de jóvenes melillenses presenta sobre el apoyo social de su comunidad en función de si se participa o no en actividades de voluntariado.

\section{MÉTODO}

\section{Participantes}

Se realizó un tipo de muestreo no probabilístico, por conveniencia, en función de la disponibilidad de los participantes. En total, 192 jóvenes de la ciudad autónoma de Melilla participaron. La edad media fue de 23.25 años $(D T=4.10)$. Por género, 58 eran hombres $(30.2 \%)$ y 134 mujeres $(69.8 \%)$. En lo relativo al nivel de estudios, el $61.5 \%$ contaba con estudios universitarios, finalizados 0 en proceso. Respecto a la religión, el $56.3 \%$ eran cristianos, el $28.1 \%$ musulmanes, el $1.5 \%$ practicaban otras religiones y el $14.1 \%$ ninguna.

52 jóvenes participaban en actividades de voluntariado (27.1\%) mientras que 140 no lo hacían (72.9\%). Entre las diferentes actividades de voluntariado señaladas por los participantes, el $42.31 \%$ colaboraba con ONG laicas (a modo de ejemplo, las asociaciones Cuenta con nosotros Melilla de enfermedades raras y TEAMA) y el $57.69 \%$ en asociaciones de voluntariado de tipo religioso (por ejemplo, Cáritas parroquial). 


\section{APOYO COMUNITARIO PERCIBIDO EN JÓVENES Y VOLUNTARIADO}

\section{Instrumento}

El Cuestionario de Apoyo Comunitario Percibido (Perceived Community Support QuestionnairePCSQ), de Herrero y Gracia (2007), fue el instrumento empleado para la recogida de la información. Se trata de un cuestionario integrado por 14 ítems, a los cuales se ha de responder según una escala tipo Likert de cinco puntos, en la cual $1=$ Muy en desacuerdo y $5=$ Muy de acuerdo. Está compuesto por tres dimensiones: integración comunitaria (ítems 1 a 4), es decir, el sentido de pertenencia y/o identificación con una comunidad o vecindario; participación comunitaria (ítems 5 a 9), la cual hace referencia al grado en el que los sujetos están implicados en actividades sociales en la comunidad; y organizaciones comunitarias (ítems 10 a 14), esto es, el grado de apoyo percibido de grupos voluntarios y organizaciones (por ejemplo, clubes recreativos y deportivos así como servicios, asociaciones políticas y cívicas en la comunidad). Según Herrero y Gracia (2007), el PCSQ posee una fiabilidad, hallada mediante el índice de consistencia interna Alfa de Cronbach, superior a .75. También una definida estructura factorial.

Se ha analizado la fiabilidad para el presente estudio del PCSQ, igualmente mediante el estadístico Alfa de Cronbach, obteniendo un valor de .85.

\section{Procedimiento}

De recogida de información.

Se accedió al alumnado universitario de la Facultad de Ciencias de la Educación y del Deporte del Campus Universitario de Melilla (Universidad de Granada) así como a diferentes asociaciones de voluntariado integradas en la ciudad autónoma de Melilla. En ambos casos, se informó de los objetivos del estudio y se solicitó la participación voluntaria y anónima de sus integrantes.

\section{De análisis estadístico de datos.}

Para determinar si existe relación entre el apoyo comunitario percibido y participar o no en actividades de voluntariado se desarrolló un estudio de tipo correlacional, empleando para ello el análisis de correlación de Pearson.

\section{RESULTADOS}

Los resultados del análisis correlacional se presentan en la Tabla 1. 
Tabla 1

Análisis de correlación de Pearson del Apoyo comunitario percibido y

la participación en actividades de voluntariado

\begin{tabular}{|c|c|c|}
\hline \multirow[b]{2}{*}{ Apoyo comunitario percibido } & \multicolumn{2}{|c|}{$\begin{array}{l}\text { Participación en actividades de } \\
\text { voluntariado }\end{array}$} \\
\hline & $r$ & $p$ \\
\hline Me identifico con mi comunidad & .027 & .737 \\
\hline Mis opiniones son valoradas por mi comunidad & $.224 * *$ & .005 \\
\hline Solo unas pocas personas de mi comunidad me conocen & -.039 & 631 \\
\hline Siento que pertenezco a mi comunidad & $.180^{*}$ & .024 \\
\hline $\begin{array}{l}\text { Colaboro en organizaciones y asociaciones en } \mathrm{mi} \\
\text { comunidad }\end{array}$ & $.403 * * *$ & .000 \\
\hline Realizo actividades en mi comunidad & $.344 * * *$ & .000 \\
\hline $\begin{array}{l}\text { Formo parte de grupos sociales o cívicos en mi } \\
\text { comunidad }\end{array}$ & $.367 * * *$ & .000 \\
\hline Respondo a las llamadas de apoyo en mi comunidad & $.339 * * *$ & .000 \\
\hline No realizo actividades socio-recreativas en mi comunidad & $-.214 * *$ & .007 \\
\hline $\begin{array}{l}\text { Podría encontrar a alguien que me ayudara a sentirme } \\
\text { mejor }\end{array}$ & -.053 & .511 \\
\hline $\begin{array}{l}\text { Encontraría a alguien que me escuchara cuando me } \\
\text { sintiera bajo de ánimo }\end{array}$ & .091 & .259 \\
\hline Encontraría una fuente de satisfacción para mí & -.011 & .895 \\
\hline $\begin{array}{l}\text { Sería capaz de animar a los demás y estar en un mejor } \\
\text { estado de ánimo }\end{array}$ & $.184 *$ & .022 \\
\hline Me relajaría y olvidaría fácilmente mis problemas & .143 & .074 \\
\hline
\end{tabular}

${ }^{*} p<.05, * * p<.01, * * * p<.001$

\section{DISCUSIÓN}

En general, se encuentran diferentes correlaciones estadísticamente significativas en la percepción que los jóvenes melillenses del estudio presentan sobre el apoyo comunitario en función de si participan 0 no en acciones relativas al voluntariado (Brissette et al., 2000; Farrel et al., 2004; Hyde \& Knowles, 2013). En particular, se hallan en dos de los cuatro ítems de la dimensión integración comunitaria, la cual integra la percepción sobre el grado de integración en la comunidad, es decir, sentido de vinculación, pertenencia o comunidad (Galen et al., 2015). Así, los jóvenes que participan en actividades de voluntariado poseen un mayor grado de identificación con su entorno social general así como valoración de sus opiniones en el mismo, desarrollando en mayor medida un sentido de pertenencia y de formar parte de la comunidad (Carpiano \& Hystad, 2011; Dalton, Elias, \& Wandersman, 2001).

Dentro del PCSQ, la dimensión donde todas las correlaciones han sido significativas ha sido la relativa a la participación comunitaria. De este modo, la percepción de ser un miembro activo de la comunidad, participando e involucrándose en la misma, correlaciona positivamente con la acción de voluntariado. La participación activa en las actividades de la comunidad lleva asociada expectativas tanto de nuevas oportunidades de interacción interpersonal como de la creación de nuevas relaciones, lo que incrementa las oportunidades de intercambio de apoyo (Hart, 2008; Kisby, 2010). Además, también posibilita una actitud más positiva hacia otros miembros de la comunidad así como un mayor sentido de la comunidad y una mayor satisfacción (Wandersman \& Florin, 2000). 


\section{APOYO COMUNITARIO PERCIBIDO EN JÓVENES Y VOLUNTARIADO}

Como también era previsible, la correlación es negativa con la no realización de actividades sociorecreativas en la comunidad.

Respecto a la dimensión organizaciones comunitarias, tan sólo se encuentra una correlación positiva en uno de los cinco ítems que la componen, "Sería capaz de animar a los demás y estar en un mejor estado de ánimo". Esta dimensión se centra en comprender el valor de la participación en términos de las necesidades de apoyo y los recursos disponibles para el individuo, es decir, el apoyo social percibido en las organizaciones comunitarias, lo cual permite comprender mejor los procesos de apoyo en la comunidad (McLaren \& Hawe, 2005).

Puesto que es importante que los jóvenes participen en diversas acciones de voluntariado (García et al., 2015; Gil et al., 2015; Hyde \& Knowles, 2013; Linsay, 2016), es necesario que se potencien campañas promocionales del voluntariado destinadas a la población juvenil que estimulen su implicación (Brewis, 2010; Grönlund et al., 2011; Holdsworth \& Brewis, 2014). De este modo, no se ofrecerá únicamente un mayor apoyo comunitario a la sociedad, en general, sino que igualmente se alcanzará un mayor bienestar psicológico por parte de las personas implicadas, en particular (Binder \& Freytag, 2013; Herrero et al., 2004; Kumar et al., 2012).

Por último, la muestra de este estudio ha sido relativamente pequeña y, además, se ha empleado solamente un instrumento de recogida de información. De cara a estudios futuros es recomendable incrementar el número tanto de participantes como de instrumentos de evaluación. Al respecto, además del cuestionario empleado, se podría diseñar alguna entrevista para obtener información en mayor profundidad. No cabe duda de que este ámbito de investigación aún tiene un largo recogido en el que se podrán determinar, en mayor medida, diferentes variables implicadas así como estrategias de intervención.

\section{REFERENCIAS BIBLIOGRÁFICAS}

Barrera, M. (2000). Social support research in community psychology. In J. Rappaport, \& E. Seidman (Eds.), Handbook of Community Psychology (pp. 215-245). New York, NY: Springer.

Binder, M., \& Freytag, A. (2013). Volunteering, subjective well-being and public policy. Journal of Economic Psychology, 34, 97-119. doi:10.1016/j.joep.2012.11.008

Brewis, G. (2010). From Service to Action? Students, Volunteering and Community Action in Mid Twentieth-Century Britain. British Journal of Educational Studies, 58(4), 439-449. doi:10.1080/00071005.2010.527668

Brissete, I., Cohen, S., \& Seeman, T. (2000). Measuring social integration and social networks. In S. Cohen, L. Underwood, \&y B. Gottlieb (Eds.), Social support measurement and intervention: $A$ guide for health and social scientists (pp. 53-85). Oxford, UK: Oxford University Press.

Carpiano, R. M., \& Hystad, P. W. (2011). "Sense of community belonging" in health surveys: What social capital is it measuring? Health \& Place, 17(2), 606-617. doi:10.1016/j.healthplace.2010.12.018

Coffey A., \& McCarthy, G.M. (2013). Older people's perception of their readiness for discharge and postdischarge use of community support and services. International Journal of Older People Nursing 8(2), 104-115. doi:10.1111/j.1748-3743.2012.00316.x

Dalton, J., Elias, M., \& Wandersman, A. (2001). Community psychology: Linking individuals and communities. Stamford, CT: Wadsworth.

Esteban-Guitart, M., Viladot, M. A., \& Giles, H. (2015). Perceived institutional support among young indigenous and mestizo students from Chiapas (México): a group vitality approach. Journal of $\begin{array}{llll}\text { Multilingual and } \quad \text { Multicultural Development, } & \text { 36(2), } & \text { 124-135. }\end{array}$ doi:10.1080/01434632.2014.898645 
Farrel, S., Aubry, T., \& Coulombe, D. (2004). Neighborhoods and neighbors: Do they contribute to personal well-being? Journal of Community Psychology, 32(1), 9-25. doi:10.1002/jcop.10082

Gadalla, T.M. (2010). The Role of Mastery and Social Support in the Association between Life Stressors and Psychological Distress in Older Canadians. Journal of Gerontological Social Work, 53(6), 512-530. doi:10.1080/01634372.2010.490691

Galen, L.W., Sharp, M., \& McNulty, A. (2015). Nonreligious Group Factors versus Religious Belief in the Prediction of Prosociality. Social Indicators Research, 122(2), 411-432. doi:10.1007/s11205-014-0700-0

García, I., Marcuello, C., \& Saz, M.I. (2015). Analysis of Volunteering Among Spanish Children and Young People: Approximation to Their Determinants and Parental Influence. Voluntas: International Journal of Voluntary and Nonprofit Organizations, 26(4), 1360-1390. doi:10.1007/s11266-014-9487-5

Gil, A. I., Marcuello, C., \& Saz, M. I. (2015). Youth Volunteering in Countries in the European Union. Approximation to Differences. Nonprofit and Voluntary Sector Quarterly, 45(5), 971-991. doi:10.1177/0899764015609731

Grönlund, H., Holmes, K., Kang, C., Cnaan, R. A., Handy, F., Brudney, J. L., ...Zrinš ak, S. (2011). Cultural Values and Volunteering: A Cross-cultural Comparison of Students' Motivation to Volunteer in 13 Countries. Journal of Academic Ethics, 9(2), 87-106. doi:10.1007/s10805-0119131-6

Guthrie, D. D., \& Fruiht, V. (2018). On-Campus Social Support and Hope as Unique Predictors of Perceived Ability to Persist in College. Journal of College Student Retention: Research, Theory \& Practice. Advance online publication. doi:10.1177/1521025118774932

Hart, R. A. (2008). Children s participation: the theory and practice of involving young citizens in community development and environmental care. New York, NY: UNICEF.

Herrero, J., \& Gracia, E. (2007). Measuring perceived community support: factorial structure, Iongitudinal invariance, and predictive validity of the PCSQ (Perceived Community Support Questionnaire). Journal of Community Psychology, 35(2), 197-217. doi:10.1002/jcop.20143

Herrero J., Meneses, J., Valiente, L., \& Rodríguez, J. (2004). Participación social en contextos virtuales. Psicothema, 16, 456-460.

Holdsworth, C., \& Brewis, G. (2014). Volunteering, choice and control: a case study of higher education student volunteering. Journal of Youth Studies, 17(2), 204-219. doi:10.1080/13676261.2013.815702

Hyde, M. K., \& Knowles, S. R. (2013). What predicts Australian university students' intentions to volunteer their time for community service? Australian Journal of Psychology, 65(3), 135-145. doi:10.1111/ajpy.12014

Kisby, B. (2010). The Big Society: Power to the People? The Political Quarterly, 81(4), 484-491. doi:10.1111/j.1467-923X.2010.02133.x

Kossek, E. E., Pichler, S. M., Meece, D., \& Barratt, M. E. (2008). Family, friend, and neighbor child care providers and maternal well-being in low-income systems: An ecological social perspective. Journal of Occupational and Organizational Psychology, 81(3), 369-391. doi:10.1348/096317908X324387

Kumar, S., Calvo, R., Avendano, M., Sivaramakrishnan, K., \& Berkman, L. F. (2012). Social support, volunteering and health around the world: Cross-national evidence from 139 countries. Social Science \& Medicine, 74(5), 696-706. doi:10.1016/j.socscimed.2011.11.017

Lakey, B., \& Orehek, E. (2011). Relational regulation theory: A new approach to explain the link between perceived social support and mental health. Psychological Review, 118(3), 482-495. 


\section{APOYO COMUNITARIO PERCIBIDO EN JÓVENES Y VOLUNTARIADO}

doi:10.1037/a0023477

Lambert, T., Casper, W. J., \& Eby, L. T. (2013). Work, family and community support as predictors of work-family conflict: A study of low-income workers. Journal of Vocational Behavior, 82(1), 59-68. doi:10.1016/j.jvb.2012.11.006

Lewis, V. A., MacGregor, C. A., \& Putnam, R. D. (2013). Religion, networks, and neighborliness: The impact of religious social networks on civic engagement. Social Science Research, 42(2), 331346. doi:10.1016/j.ssresearch.2012.09.011

Linsay, S. (2016). A scoping review of the experiences, benefits, and challenges involved in volunteer work among youth and young adults with a disability. Disability and Rehabilitation, 38(16), 1533-1546. doi:10.3109/09638288.2015.1107634

Mancini, V. O., Rigoli, D., Heritage, B., Roberts, L. D., \& Piek, J. P. (2016). The Relationship between Motor Skills, Perceived Social Support, and Internalizing Problems in a Community Adolescent Sample. Frontiers in Psychology, 7, 543. doi:10.3389/fpsyg.2016.00543

McDonald, C., Wall, K., Corwin, D., Brooks, C., y Koopman, C. (2013). The perceived effects of psycho-spiritual integrative therapy and community support groups on coping with breast cancer: a qualitative analysis. European Journal for Person Centered Healthcare, 1(2), 298-309.

McLaren, L., \& Hawe, P. (2005). Ecological perspectives in health research. Journal of Epidemiology and Community Health, 59(1), 6-14. doi:10.1136/jech.2003.018044

Melchiorre, M. G., Chiatti, C., Lamura, G., Torres-Gonzales, F., Stankunas, M., Lindert, J., ...Soares, J. F .J. (2013). Social Support, Socio-Economic Status, Health and Abuse among. Older People in Seven European Countries. PLOS ONE, 8(1), e54856. doi:10.1371/journal.pone.0054856

Moritsugu, J., Vera, E., Wong, F. Y., \& Grover, K. (2016). Community Psychology (5 ${ }^{\text {th }}$ ed.). New York, NY: Routledge.

Musick, M.A., y Wilson, J. (2007). Volunteers: A Social Profile (1st ed.). Bloomington: Indiana University Press.

Ng, E. C. W., Chan, C. C., \& Lai, M. K. (2014). Hope and life satisfaction among underprivileged children in Hong Kong: the mediating role of perceived community support. Journal of Community Psychology, 42(3), 352-364. doi:10.1002/jcop.21614

Oberle, E., Schonert-Reichl, K. A., \& Zumbo, B. D. (2011). Life Satisfaction in Early Adolescence: Personal, Neighborhood, School, Family, and Peer Influences. Journal of Youth and Adolescence, 40(7), 889-901. doi:10.1007/s10964-010-9599-1

Patwardhan, I., Mason, W. A., Savolainen, J., Chmelka, M. B., Miettunen, J., \& Järvelin, M. R. (2017). Childhood cumulative contextual risk and depression diagnosis among young adults: The mediating roles of adolescent alcohol use and perceived social support. Journal of Adolescence, 60, 16-26. doi:10.1016/j.adolescence.2017.07.008

Paxton, P., Reith, N. E., \& Glanville, J. L. (2014). Volunteering and the Dimensions of Religiosity: A Cross-National Analysis. Review of Religious Research, 56(4), 597-625. doi:10.1007/s13644014-0169-y

Rappaport, J., \& Seidman, E. (Eds.) (2000). Handbook of Community Psychology. New York, NY: Springer.

Snapp, S. D., Watson, R. J., Russell, S. T., Diaz, R. M., \& Ryan, C. (2015). Social Support Networks for LGBT Young Adults: Low Cost Strategies for Positive Adjustment. Family Relations. Interdisciplinary Journal of Applied Family Science, 64(3), 420-430. doi:10.1111/fare.12124

Stroope, S. (2011). Education and religion: Individual, congregational, and cross-level interaction effects on biblical literalism. Social Science Research, 40(6), 1478-1493. doi:10.1016/j.ssresearch.2011.05.001 
Wandersman, A., \& Florin, P. (2000). Citizen participation and community organizations. In J. Rappaport \& E. Seidman (Eds.), Handbook of community psychology (pp. 247-272). New York, NY: Kluwer/Plenum.

Wang, J. L., Hsieh, H. F., Assari, S., Gaskin, J., \& Rost, D. H. (2018). The Protective Effects of Social Support and Engagement Coping Strategy on the Relationship between Perceived Discrimination and Psychological Distress among Chinese Migrant Children. Youth \& Society, 50(5), 593-614. doi:10.1177/0044118X15619804 
